\title{
Dynamic behavior of structural-acoustic systems in feedforward control of sound radiation
}

\author{
Ricardo A. Burdisso and Chris R. Fuller \\ Department of Mechanical Engineering, Virginia Polytechnic Institute and State University, Blacksburg, \\ Virginia 24061-0238
}

(Received 9 May 1991; revised 6 November 1991; accepted 9 December 1991)

\begin{abstract}
The dynamic characteristics of a beam under active control to minimize the coupled sound radiation is investigated. The beam is subjected to a harmonic point force and the resulting radiated field minimized by utilizing a piezoceramic actuator as control input. The error information from the acoustic medium is obtained by placing a single microphone in the acoustic far field. A feedforward control approach is used to derive the optimum control input applied to the actuator. The analysis shows that when the error microphone is placed along a line perpendicular to the radiating surface, the controlled structure has new eigenvalues and eigenfunctions. The controlled system eigenproperties are only a function of the control force location and independent of the disturbance input. A numerical example demonstrates the applicability of the formulation. The numerical results are also corroborated experimentally using a feedforward controller implemented on a TMS320C20 DSP processor.
\end{abstract}

PACS numbers: $43.40 . \mathrm{Vn}, 43.40 . \mathrm{Cw}$

\section{INTRODUCTION}

Sound radiated by vibrating structures is a persistent problem in numerous industrial applications, and it has long been a subject of research interest in the acoustic community. The common practice to attentuate the noise field involves the redesign of the radiating structure. However, this passive technique often results in a much heavier system due to an increase in mass and it may not always be effective at low frequencies. In recent years, considerable effort has been devoted to develop techniques for active control of low-frequency structurally radiated sound. The traditional approach of active noise control relies on the use of acoustic control sources. Successful implementations of this technique in one-dimensional sound fields have been reported in the literature. ${ }^{1,2}$ Active control of structurally radiated three-dimensional sound fields by means of acoustic sources have been investigated by Deffayet and Nelson. ${ }^{3}$ This work demonstrated that for distributed noise sources, as in the case of structural noise, multiple channels of acoustic control inputs are necessary if global attenuation of the sound field is desired, particularly at higher frequencies.

To reduce the dimensionality of the controller, a new active control approach has been proposed by Fuller ${ }^{4}$ in which the control inputs are applied directly to the vibrating structure. Experimental and analytical investigations of sound transmission and radiation from harmonically excited panels ${ }^{5,6}$ have shown that global attenuation can be achieved with only one or two control inputs. In conjunction with this concept, the feedforward control algorithm used in these investigations and others ${ }^{7,8}$ have demonstrated the potential of this combined approach in active control of structurally radiated sound under stationary disturbances. In feedforward control, the undesirable noise field is canceled by superimposing a secondary sound field produced by the control in- puts and coherent with the noise disturbance. The proper excitation to be applied to the control inputs is derived from error information sensed directly from the acoustic medium to be minimized. This results in a forced modification of the structural response such that the dominant radiating modes of the structure are reduced (modal suppression) or the residual response is not attenuated but behaves as a much less efficient radiator (modal restructuring). ${ }^{8}$

The dynamic characteristics of feedforward controlled elastic structures in vibration suppression have recently been investigated by Burdisso and Fuller. ${ }^{9,10}$ They have shown for a single-input, single-output (SISO) system that the controlled system has new eigenproperties, and that instabilities in the feedforward algorithm occurs at the controlled system natural frequencies. The main aim of this paper is to extend this analysis when feedforward active control techniques are used for attenuating structurally radiated sound fields. To this end, the analysis from Ref. 9 is applied to the active control of sound radiated from the baffled simply supported beam of Fig. 1. The beam is excited by a harmonic point force and the radiated sound field is sensed by a microphone placed in the acoustic field. The control source is a piezoceramic pair mounted on each side of the beam surfaces and wired out-of-phase such that the forcing function is equivalent to a pair of bending moments. ${ }^{11-13}$ The control input is obtained by feeding forward fully coherent information obtained from the same noise disturbance through the compensator or controller $G(\omega)$. The compensator, that gives the optimum complex amplitude to the control input, is determined so as to minimize the mean square of the sound pressure at the microphone location.

The analysis presented here gives insight into this problem and shows that the controlled system (i.e., the structural-acoustic system) effectively behaves as having new properties. It also contributes to the design of "smart" struc- 


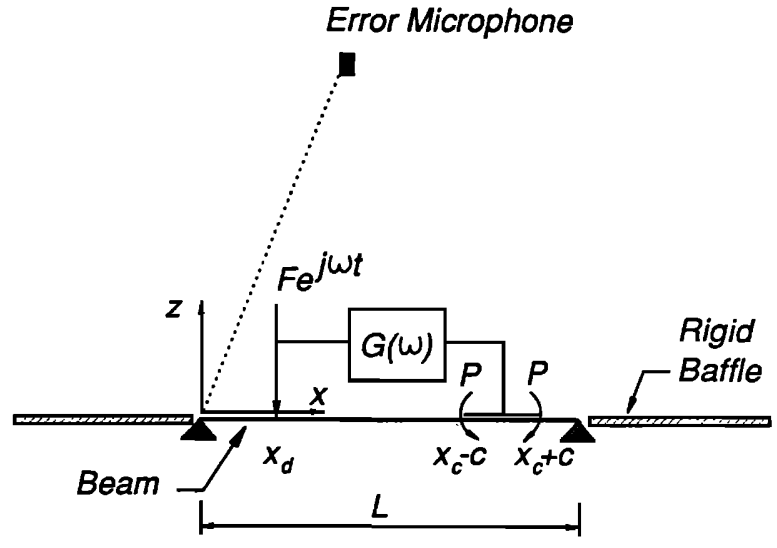

FIG. 1. Feedforward control approach for simply supported beam.

tures with embedded sensors and actuators. In many applications, the placement of an error microphone in the acoustic medium is impractical or simply not possible. Thus the development and design of structural sensors to replace the error microphone is sometimes desired. In order to have a properly designed structural sensor equivalent to a microphone in the acoustic field, the controlled system dynamics with the use of an error microphone must be first studied and understood in detail.

\section{BEAM RESPONSE AND RADIATED FAR-FIELD PRESSURE}

The response of a beam subjected to a harmonic input can be given as a linear combination of the modes as follows:

$$
w(x, t)=\sum_{n=1}^{N} q_{n}(\omega) \phi_{n}(x) e^{j \omega t},
$$

where $q_{n}(t)$ is the $n$th generalized coordinate, $\phi_{n}(x)$ is the $n$th eigenfunction, and $N$ is the number of modes included in the analysis. The excitation frequency is $\omega$ and $j$ is the imaginary number. The generalized coordinate or modal displacement $q_{n}(\omega)$ is given as

$$
\begin{aligned}
q_{n}(\omega) & =H_{n}(\omega) \phi_{n}\left(x_{d}\right) F \\
& =\left(\omega_{n}^{2}-\omega^{2}+2 j \eta_{n} \omega_{n} \omega\right)^{-1} \phi_{n}\left(x_{d}\right) F,
\end{aligned}
$$

where $H_{n}(\omega)$ is the $n$th modal frequency response function (FRF); $\omega_{n}$ and $\eta_{n}$ are the $n$th natural frequency and modal damping ratio, respectively; and $F$ is the amplitude of the disturbance force applied at $x_{d}$. Equation (2) assumes the mode shape to be normalized with respect to the mass distribution, $m(x)$. That is, they satisfy the orthogonality condition

$$
\int_{0}^{L} \phi_{n}(x) m(x) \phi_{m}(x) d x=\delta_{n m},
$$

where $\delta_{n m}$ is the Kronecker delta function and $L$ is the beam length.

The beam response due to the input force $F$ is then assumed to be controlled by the secondary load, given by a bending moment pair, with complex amplitude $P$. Then, by the superposition principle, the combined or total system response becomes

$$
w^{c}(x, t)=\sum_{n=1}^{N} q_{n}^{c}(\omega) \phi_{n}(x) e^{j \omega t}
$$

where $q_{n}^{c}(\omega)$ is the $n$th modal displacement given by

$$
q_{n}^{c}(\omega)=\left[\phi_{n}\left(x_{d}\right) F+p_{n} P\right] H_{n}(\omega) .
$$

The control modal force component $p_{n}$ can be shown to be given by

$$
p_{n}=\frac{d \phi_{n}\left(x_{c}+c\right)}{d x}-\frac{d \phi_{n}\left(x_{c}-c\right)}{d x},
$$

where $d \phi_{n}(x) / d x$ is the slope of the mode shape, $x_{c}$ is the coordinate of the middle point of the piezoceramic actuator, and $2 c$ is the actuator length.

The optimum complex amplitude of the control input $P$ is obtained so as to minimize the radiated far-field pressure in the acoustic medium. Thus, an expression to compute the far-field pressure radiated by the beam is first required. The far-field pressure radiated by a vibrating surface at a point in the acoustic field is given by the Rayleigh integral ${ }^{14}$ as follows:

$$
p(\mathbf{r}, t)=\frac{j \omega \rho}{2 \pi} \int_{S} \dot{w}^{c}\left(\mathbf{r}_{s}\right) \frac{e^{-j k R}}{R} d S,
$$

where, as shown in Fig. 2, $\mathrm{r}$ is the position vector of the observation point; $\mathbf{r}_{s}$ is the position vector of the elemental surface $d S$ having normal velocity $\dot{w}^{c}\left(\mathbf{r}_{s}\right) ; R$ is the magnitude of the vector difference $\mathbf{r}-\mathbf{r}_{s} ; \rho$ is the fluid density; and $k$ is the acoustic wave number given as $k=\omega / c$, where $c$ is the speed of sound in the fluid. Here, it is assumed that the acoustic medium is air and thus no feedback of the fluid motion into the structure takes place.

The normal velocity $\dot{w}^{c}$ is found by differentiating Eq. (4) which is in turn substituted into Eq. (7). Then, assuming the observation point to be in the far-field $(R \gg L)$, Eq. (7) can be approximated as follows: ${ }^{14}$

$$
\begin{aligned}
p(r, \theta, \psi, t)= & \sum_{n=1}^{N} j \omega q_{n}^{c}(\omega) \frac{j \omega \rho}{2 \pi} \frac{e^{-\jmath k r}}{r} \int_{0}^{b} \int_{0}^{L} \phi_{n}(x) \\
& \times \exp \left[-j\left(\alpha \frac{x}{L}+\beta \frac{y}{b}\right)\right] d x d y e^{(\omega t},
\end{aligned}
$$

where $(r, \theta, \psi)$ are the polar coordinates of the observation point as shown in Fig. 2, $b$ is the beam width and

$$
\begin{aligned}
& \alpha=k L \sin \theta \cos \psi \\
& \beta=k b \sin \theta \sin \psi
\end{aligned}
$$

Closed form solution of the integral in Eq. (8) can be obtained for simply supported beams and plates where the eignefunctions are expressed in terms of sine functions. ${ }^{15,16}$ Then, the pressure at the far field can be written as

$$
\begin{aligned}
p(r, \theta, \psi, t)= & \frac{j \omega \rho}{2 \pi} \frac{e^{-j k r}}{r} \sum_{n=1}^{N}\left\{j \omega \left[\phi_{n}\left(x_{d}\right) F\right.\right. \\
& \left.\left.+p_{n} P\right] H_{n}(\omega)\right\} \xi_{n}(\omega, \alpha, \beta) e^{j \omega t},
\end{aligned}
$$

where $\xi_{n}(\omega, \alpha, \beta)$ is the integral in Eq. (8). As an illustrative example, the mode shape of uniform simply supported beam $\phi_{n}(x)=(2 / m L)^{-1 / 2} \sin (n \pi x / L)$ renders the function $\xi_{n}(\omega, \alpha, \beta)$ to be as follows: ${ }^{15}$ 


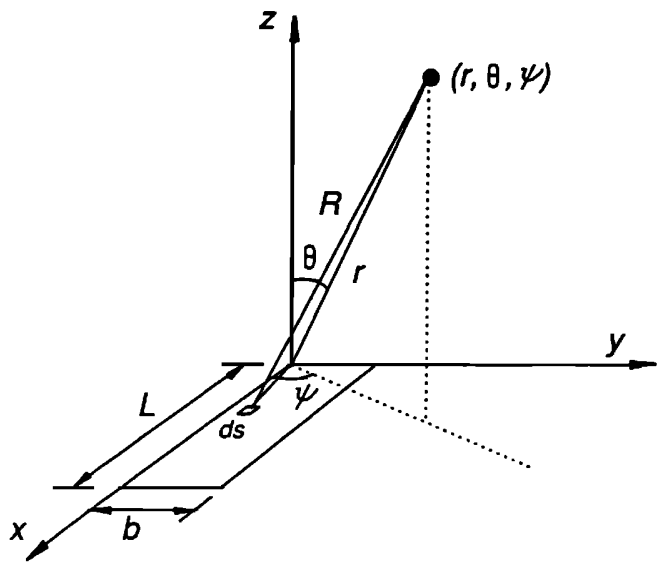

FIG. 2. System arrangement and coordinate system.

$$
\xi_{n}(\omega, \alpha, \beta)=b L\left(\frac{2}{m L}\right)^{1 / 2} n \pi \frac{1-(-1)^{n} e^{j \alpha}}{(n \pi)^{2}-\alpha^{2}} \frac{1-e^{j \beta}}{\beta} .
$$

The function $\xi_{n}(\omega, \alpha, \beta)$, as seen in Eq. (11), is a frequency-dependent complex variable. This variable can be considered as an influence function that relates the $n$th modal displacement to the sound pressure at the observation point. That is, the sound-pressure magnitude and phase at the observation point due to a unit amplitude of the $n$th modal coordinate is related to the magnitude and phase of $\xi_{n}(\omega, \alpha, \beta)$. Herein, we will refer to this function as the modal acoustic influence function.

From Eq. (11), it is noted that both the magnitude and phase of $\xi_{n}(\omega, \alpha, \beta)$ are frequency dependent, and thus the phase delay between the modal coordinate and the pressure at the observation point depends on the excitation frequency $\omega$. However, if the observation point lies along the $z$ axis in Fig. $2(\theta=0)$, the quantities $\alpha$ and $\beta$ vanish and renders $\xi_{n}(\omega, 0,0)=\xi_{n}$ independent of the excitation frequency. This implies that the sound pressure along this line is in phase with the modal response, and this will have an important effect in the dynamics of the controlled system as shown in later sections.

Hence, the beam response is given by Eq. (4) while the far-field-radiated pressure is expressed by Eq. (10). From these two equations the optimum complex amplitude $P$ to minimize the radiated sound pressure can now be derived as presented in the following section.

\section{OPTIMUM CONTROL FORCE}

In feedforward control, the optimum complex amplitude of the control force $P$ is defined so as to minimize a quadratic cost function of a measurable response quantity. Here, the radiated sound pressure is sensed by placing an error microphone in the far field with polar coordinates $\left(r_{e}, \theta_{e}, \psi_{e}\right)$. Then, the optimum control complex amplitude $P$ is obtained by minimizing the mean-square value of the pressure at the error microphone location. The mean-square pressure is obtained as

$$
C(P)=p\left(r_{e}, \theta_{e}, \psi_{e}, t\right) p^{*}\left(r_{e}, \theta_{e}, \psi_{e}, t\right)
$$

where an asterisk $(*)$ over a complex quantity denotes it's complex conjugate.

This cost function is a positive definite function with respect to $P$, and therefore there exists only one minimum. By differentiating Eq. (12) with respect to the real and imaginary part of the control force $P$ and setting to zero yields the optimum $P$ as

$P=-G(\omega) F=-\frac{\sum_{n=1}^{N} H_{n}(\omega) \phi_{n}\left(x_{n}\right) \xi_{n}\left(\omega, \alpha_{e}, \beta_{c}\right)}{\sum_{n=1}^{N} H_{n}(\omega) p_{n} \xi_{n}\left(\omega, \alpha_{c}, \beta_{c}\right)} F$,

where $\alpha_{e}$ and $\beta_{e}$ are Eq. (9) evaluated at the error sensor coordinate $\left(r_{e}, \theta_{e}, \psi_{e}\right)$.

Equation (13) defines the controller $G(\omega)$ as the ratio of two functions. The numerator is related to the transfer function between the disturbance force load and the pressure at the error microphone, whereas the denominator is related to the transfer function between the control load and the pressure at the error microphone. If the error microphone is placed in the acoustic field such that $\xi_{k}(\omega, \alpha, \beta)$ vanishes, this implies that the $k$ th mode is unobservable, and thus, the summation terms in Eq. (13) are only over the observable modes. Similarly, if the control load is such that $p_{k}$ is zero the $k$ th mode is uncontrollable and the summation term in the denominator is over the controllable modes only. For the clarity in the presentation, it is assumed here that all modes are observable and controllable.

Embedded into the controller is both the dynamics of the structure through the modal FRF $H_{n}(\omega)$ and the dynamics of the fluid media through the modal acoustic influence function, $\xi_{n}(\omega, \alpha, \beta)$. Thus the controlled system response will also be influenced by the dynamics of the acoustic medium. Then, replacing Eq. (13) into (5) and this into (4) gives the response of the controlled system as

$$
w^{c}(x, t)=\sum_{n=1}^{N}\left[\phi_{n}\left(x_{d}\right)-p_{n} G(\omega)\right] H_{n}(\omega) \phi_{n}(x) F e^{j \omega t}
$$

The uncontrolled system response is simply obtained from Eq. (14) by setting $G(\omega)$ to zero.

In order to underscore the main purpose of this work, the general behavior of the uncontrolled and controlled beam of Fig. 1 are presented in Figs. 3 and 4. The error microphone is placed in the far-field perpendicular to the beam $\left(\theta_{e}=\psi_{e}=0\right)$, and the beam parameters are given in Sec. IV, where the system response is analyzed in detail. Figures 3(a) and 4(a) show, respectively, the uncontrolled and controlled beam response in terms of the excitation frequency. Their associated radiation directivity in the $x-z$ plane, in dB scale, are shown in Figs. 3(b) and 4(b) for the uncontrolled and controlled system, respectively, versus frequency. It is very clear that the dynamic behavior of the controlled system differs significantly from the uncontrolled one, and, consequently, the resulting radiation. Figure 3 (b) shows large sound radiation levels of a global nature when the uncontrolled system is driven at its natural frequencies. 

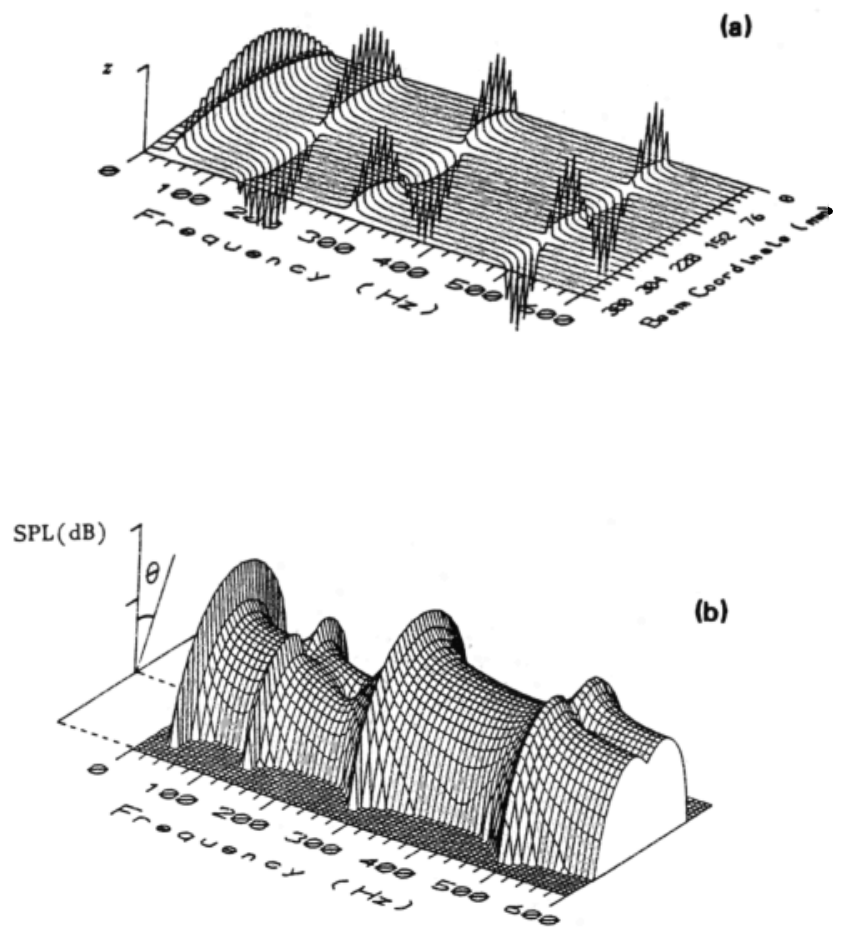

FIG. 3. Uncontrolled (a) beam displacement, and (b) radiation directivity in $x-z$ plane versus frequency.

On the other hand, Fig. 4(a) shows that the controlled beam presents resonant behavior at different frequencies from the beam with no control. The far-field radiation in Fig. 4(b) first shows the sound in the direction of error microphone is completely canceled at all frequencies. This is indicated by the notch in radiation pattern at an angle perpendicular to the beam plane $x-y$. Second, global attenuation of radiated sound cannot be achieved at frequencies where the controlled beam exhibits resonant behavior. Thus, for a particular feedforward control system design, as defined by the control input and error microphone placement, global attenuation of the acoustic field is possible only over some frequency ranges. For example, the control design in Fig. 2 achieves global attenuation of radiated sound in the ranges $0-100 \mathrm{~Hz}$ and $180-440 \mathrm{~Hz}$. Thus it is important to develop a formulation to predict the dynamics of the system under active control. This is investigated in the next sections.
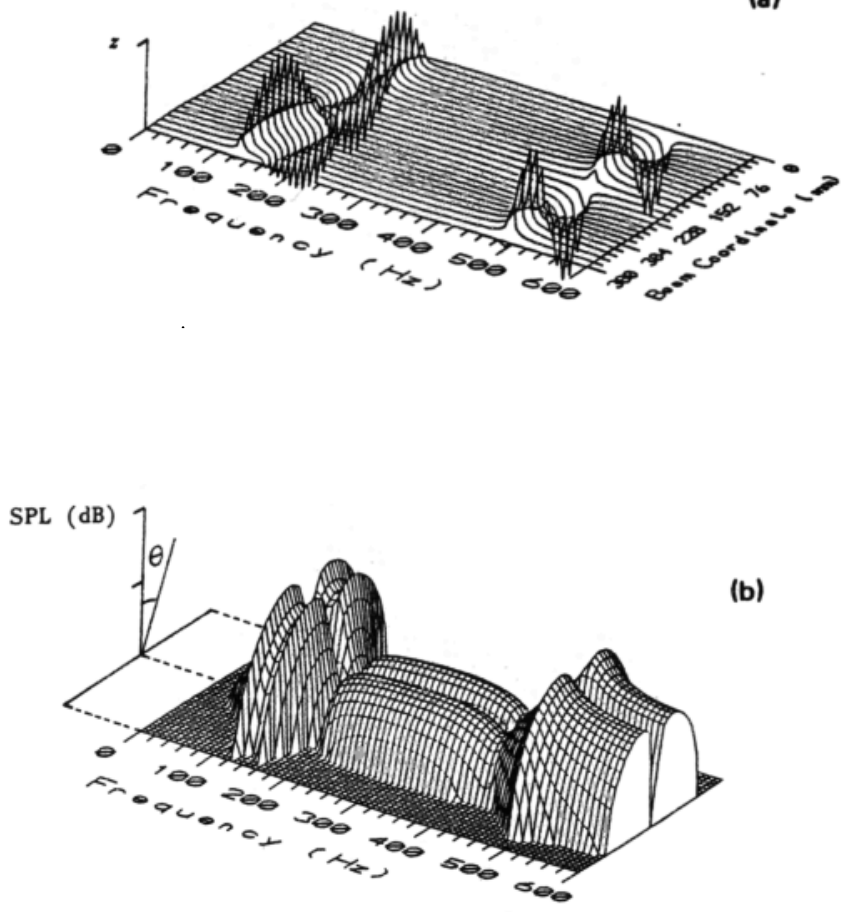

FIG. 4. Controlled (a) beam displacement, and (b) radiation directivity in $x-z$ plane versus frequency.

\section{DYNAMIC BEHAVIOR OF CONTROLLED SYSTEM}

The dynamic characteristics of the controlled beam will now be investigated. For this analysis, we assume the system to be undamped although the introduction of damping is straightforward. The controller $G(\omega)$ is first expanded by multiplying and dividing by the product of the FRF as follws:

$$
\begin{aligned}
G(\omega) & =-\frac{N(\omega)}{D(\omega)} \\
& =-\frac{\sum_{m=1}^{N} \phi_{m}\left(x_{d}\right) \xi_{m}\left(\omega, \alpha_{e}, \beta_{e}\right) \prod_{i=1}^{N}\left(\omega_{s}^{2}-\omega^{2}\right)}{\sum_{m=1}^{N} p_{m} \xi_{m}\left(\omega, \alpha_{e}, \beta_{e}\right) \prod_{\substack{i=1 \\
i \neq m}}^{N}\left(\omega_{s}^{2}-\omega^{2}\right)}
\end{aligned}
$$

Replacing Eq. (15) into (5) and after some straightforward manipulation the controlled modal amplitude is given by

$$
q_{n}^{c}(\omega)=\frac{\sum_{m \rightarrow n}^{N}\left[\phi_{n}\left(x_{d}\right) p_{m}-\phi_{m}\left(x_{d}\right) p_{n}\right] \xi_{m}\left(\omega, \alpha_{e}, \beta_{e}\right) \Pi_{i, n, m}^{N}\left(\omega_{s}^{2}-\omega^{2}\right)}{\sum_{m=1}^{N} p_{m} \xi_{m}\left(\omega, \alpha_{e}, \beta_{e}\right) \Pi_{\substack{v=1, m \\ i=m}}^{N}\left(\omega_{s}^{2}-\omega^{2}\right)}
$$

Equation (16) shows that the generalized modal coordinate of the controlled system is given by the ratio of two complex functions of frequency. The function $D(\omega)$, in the denominator of Eqs. (15) and (16), is common to all the controlled modal coordinates. Thus the dynamic behavior of the controlled system is strongly influenced by the function $D(\omega)$. By studying the features of this complex function, enough information regarding the dynamics of the controlled system can be derived. For example, the zeros of $D(\omega)$ are the poles of the controlled system, and the structural response will be unbounded if the system is driven at a system pole frequency (with no damping). The associated sound radiation will also be unbounded even though the sound pressure at the error microphone vanishes. It should be mentioned that due to the frequency-dependent term $\xi_{m}\left(\omega, \alpha_{e}, \beta_{e}\right)$ in Eq. (16), the number of poles in $D(\omega)$ are not known a priori. 
A special case, however, occurs when $\alpha_{e}$ and $\beta_{e}$ vanish. As mentioned before, in this situation the modal acoustic influence function is frequency independent and the dynamic characteristics of the controlled system can be further investigated. The rest of this paper concentrates on this particular case. Then, Eq. (16) can be written as

$$
=\frac{\boldsymbol{\Sigma}_{n}^{c}(\omega)}{\sum_{m \neq n}^{N}\left[\phi_{n}\left(x_{d}\right) p_{m}-\phi_{m}\left(x_{d}\right) p_{n}\right] \xi_{m} \Pi_{i \neq n, m}^{N}\left(\omega_{s}^{2}-\omega^{2}\right)},
$$

where $\xi_{m}$ is now a constant. Again as an illustrative example, for a uniform simply supported beam, $\xi_{m}$ becomes

$$
\xi_{m}=b L\left(\frac{2}{m L}\right)^{1 / 2} \frac{1-(-1)^{m}}{m \pi} .
$$

The numerator and denominator in Eq. (17) become polynomials in $\omega^{2}$ of order $(N-2)$ and $(N-1)$, respectively. Thus there are $(N-2)$ zeros and $(N-1)$ poles in the controlled system, and by using partial fraction expansion, as shown in Appendix A, the controlled modal coordinate can now be written as follows:

$$
q_{n}^{c}(\omega)=\sum_{l=1}^{N-1} \gamma_{c l} \frac{1}{\left(\omega_{c l}^{2}-\omega^{2}\right)} F=\sum_{l=1}^{N-1} \gamma_{l n} H_{c l}(\omega) F,
$$

where $H_{c l}(\omega)$ represents the FRF of a single degree-of-freedom (DOF) oscillator with associated natural frequency $\omega_{c l}$ which is the $l$ th pole of the controlled system. Thus, the $n$th modal coordinate of the controlled sytem is now the linear contribution of the response of $(N-1)$ single DOF oscillators.

Replacing Eq. (19) into (4) and after interchanging the position of the summation terms, the controlled system response becomes

$$
w^{c}(x, t)=\sum_{l=1}^{N-1}\left[\sum_{n=1}^{N} \gamma_{l n} \phi_{n}(x)\right] H_{c l}(\omega) F e^{j \omega t},
$$

where the term in brackets is a function of $x$ multiplied by the FRF of the controlled system. Thus, Eq. (20) can be rewritten in the typical form found in modal analysis. ${ }^{15}$ That is,

$$
w^{c}(x, t)=\sum_{l=1}^{N-1} \frac{\phi_{c l}\left(x_{d}\right)}{m_{c l}} \phi_{c l}(x) H_{c l}(\omega) F e^{j \omega t},
$$

where

$$
\frac{\phi_{c l}\left(x_{d}\right)}{m_{c l}} \phi_{c l}(x)=\sum_{n=1}^{N} \gamma_{l n} \phi_{n}(x) .
$$

Equation (21) indicates that the controlled system response is now expressed in terms of a new set of $(N-1)$ eigenfunctions $\phi_{c l}(x)$ with associated natural frequencies $\omega_{c l}$, and the modal mass is $m_{c l}$. The new mode shapes are computed by assuming them to be normalized with respect to the mass distribution of the uncontrolled beam. That is,

$$
\int_{0}^{L} \phi_{c l}(x) m(x) \phi_{c l}(x) d x=1 .
$$

Replacing Eq. (22) into (23) and using the orthogonality condition of the uncontrolled mode shapes in Eq. (3) gives

$$
\phi_{c l}(x)=\Delta \sum_{n=1}^{N} \frac{p_{n}}{\left(\omega_{c l}^{2}-\omega_{n}^{2}\right)} \phi_{n}(x),
$$

where

$$
\Delta=\sum_{n=1}^{N} \frac{p_{n}^{2}}{\left(\omega_{c l}^{2}-\omega_{n}^{2}\right)^{2}} .
$$

The analysis presented here demonstrates that when the error microphone is placed perpendicular to the radiating surface, the controlled system has new eigenvalues and eigenproperties. The controlled system eigenproperties are only functions of the control force and error microphone location and independent of the disturbance load. When the error microphone is located elsewhere, the function $D(\omega)$ is no longer a polynomial in $\omega^{2}$ and the controlled modal coordinate cannot be expanded as the linear contribution of the response of $(N-1)$ single DOF oscillators as in Eq. (19). However, again some insight into its dynamics can be gained by investigating the denominator of the controller in Eq. (13), and this is the subject of future research.

The formulation presented can also contribute to the design of a structural sensor to replace the error microphone. For a structural sensor to be equivalent to an error microphone within a frequency range, its $m$ th modal influence function should be identical to the acoustical modal influence function $\xi_{m}\left(\omega, \alpha_{e}, \beta_{e}\right)$ in that frequency range. As described before, its magnitude and phase are functions of the excitation frequency. However, any measurable structural response quantity, such as acceleration, velocity, strain, etc., will always result in a frequency-independent modal influence function. Thus any structural sensor cannot be completely equivalent to an error microphone, unless proper adaptation of the sensor's properties with frequency is possible. The physical interpretation of this limitation lies in the fact that a structural sensor cannot model the dynamics of the acoustic medium. An exception occurs when the error microphone is along a direction perpendicular to the beam surface. In this case, the acoustic influence function is frequency independent and an equivalent structural sensor is easily realizable.

\section{NUMERICAL AND EXPERIMENTAL RESULTS}

The applicability of the formulation presented here is demonstrated for the uniform beam of Fig. 1. This example problem was analytically studied and experimentally verified. The experimental rig consists of a thin steel beam mounted in a heavy rigid frame by shims attached to the edge of the beam at its ends. The beam has bending stiffness $\mathrm{EI}=5.305 \mathrm{NT} \mathrm{m}^{2}$, mass per unit length $m=0.6368 \mathrm{NT} \mathrm{s}^{2} /$ $\mathrm{m}$, length $L=0.38 \mathrm{~m}$, and width $b=0.04 \mathrm{~m}$. The system is assumed to have $0.1 \%$ damping in all the modes and it is incorporated to keep the analytical response bounded. Ten modes are included in the analysis, $N=10$. The beam is excited by a concentrated force located at $x_{d}=0.055 L$ applied by a Ling mini-shaker attached point-wise to the beam by a sting, and a Kistler force transducer was used to monitor the shaker force. The control input is a pair of bending moments applied at $x_{c l}=0.78 L$ and $x_{c 2}=0.73 L$, applied by a pair of piezoceramics patches mounted on each side on 
the beam surface and wired out-of-phase. Two Brüel \& Kjær mini-accelerometers were also mounted on the beam to measure the response. The experimental rig was placed in a large baffle located in an anechoic chamber with cutoff frequency of $200 \mathrm{~Hz}$. The sound radiated by the beam was sensed by a circular array of 13 microphones at a radius of $1.7 \mathrm{~m}$. A Brüel \& Kjær microphone placed perpendicular to the beam, with polar coordinate $\left(r=4.45 L, \theta_{e}=0, \psi_{e}=0\right)$, was used as error sensor.

After attaching the shaker and transducers, the natural frequencies of the structure were experimentally determined. Due to the sensitivity of the acoustic far field to the mode shapes, the shaker-force transducer system and the end shims were modeled in the analytical model as a point mass attached at the shaker drive point and bending springs acting at the beam ends, respectively. The analytical natural frequencies were approximated to the experimental values by adjusting the value of the concentrated mass and of the springs constant. The value of the added point mass is 0.017 $\mathrm{N} \mathrm{s}^{2} / \mathrm{m}^{2}$ and the value of the end spring stiffness is $3.05 \mathrm{NT} /$ $\mathrm{rad}$. The assumed mode method ${ }^{17}$ was used to solve for the eigenvalue problem using as admissible functions the normalized mode shapes of the uniform simply supported beam as shown in Appendix B. Excellent agreement between the experimentally measured and computed natural frequencies is found, as seen in Table I.

To compare the theoretical versus experimental control results, an adaptive feedforward control system was implemented. The block diagram of this control system is given in Fig. 5. The control approach was based upon the "filtered$X$ " version of the LMS algorithm as described in Refs. 18 and 19. The algorithm was coded in assembly language and downloaded on a PC based TMS320C20 digital signal processor. As can be seen in Fig. 5, the control system adapts the coefficients of a finite impulse response (FIR) control filter based upon the error microphone signal $e(t)$ and the $x(t)$ signal filtered by the transfer function between the control input and error microphone $T_{c c}$. Since the noise signal is a pure tone sinusoidal, only two coefficients were used in the FIR filter. The reference signal was taken from the same function generator as the noise signal, thus providing a coherent control signal with the disturbance.

TABLE I. Simply supported beam natural frequencies.

\begin{tabular}{|c|c|c|}
\hline \multirow[b]{2}{*}{ Mode } & \multicolumn{2}{|c|}{ Natural frequency $[\mathrm{Hz}]$} \\
\hline & Analytical & Experimenta \\
\hline 1 & 32.66 & 32 \\
\hline 2 & 125.94 & 128 \\
\hline 3 & 279.07 & 279 \\
\hline 4 & 489.57 & 491 \\
\hline 5 & 756.32 & 753 \\
\hline 6 & 1080.39 & 1120 \\
\hline 7 & 1467.80 & 1486 \\
\hline 8 & 1922.46 & $\cdots$ \\
\hline 9 & 2447.48 & $\cdots$ \\
\hline 10 & 3046.79 & $\cdots$ \\
\hline
\end{tabular}

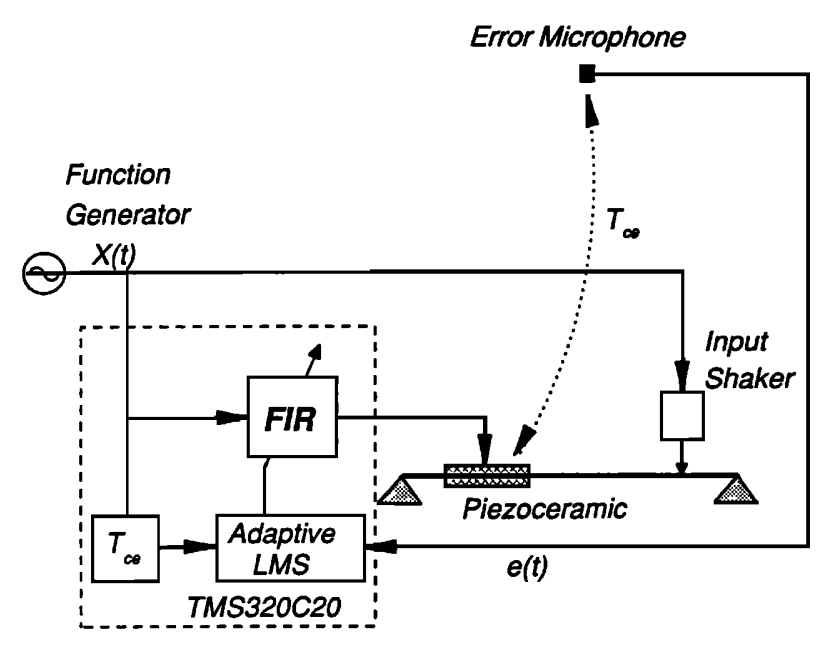

FIG. 5. Time domain filtered- $x$ LMS control block diagram.

The dynamic behavior of the beam is illustrated by plotting the response at the input force location with and without control. Assuming a unit magnitude of the input force $F$, Fig. 6 shows the amplitude and phase of the acceleration at $x_{d}$ as a function of the excitation frequency $\omega$. The dashed line is the response of the uncontrolled beam obtained by setting $P$ to zero in Eq. (14), while the continuous line corresponds to the controlled system calculated from Eqs. (13) and (14). The response of the uncontrolled beam shows resonance peaks at the frequencies given in Table I. On the other hand, when the control force $P$ is applied, the response shows resonance behavior at excitation frequencies near 120,160, 480, 900, and $1200 \mathrm{~Hz}$, respectively. The computed phase angle,
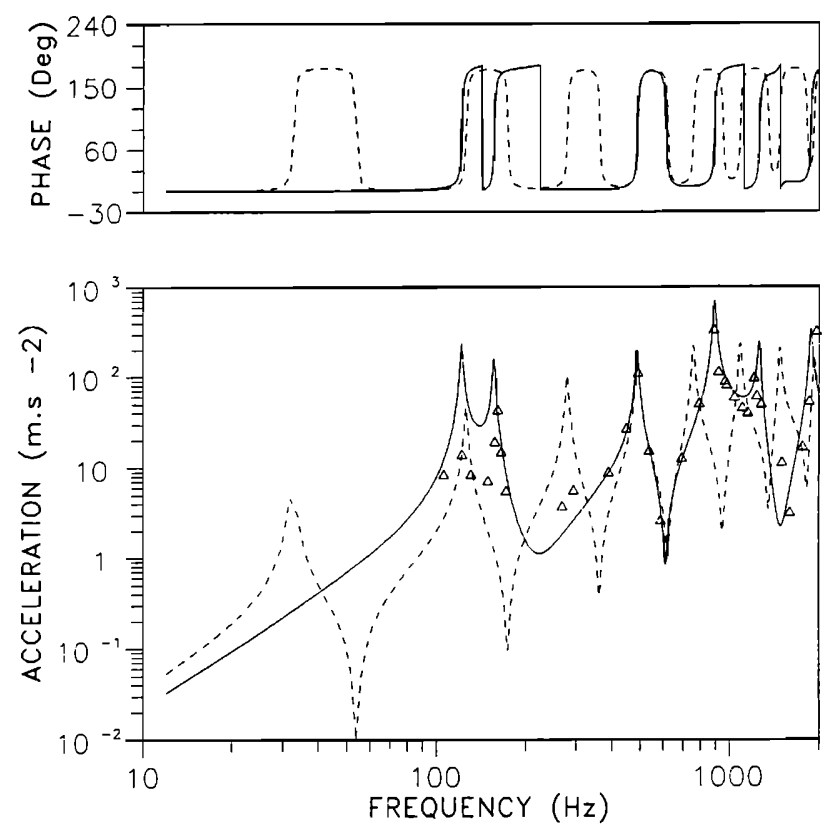

FIG. 6. Acceleration at disturbance force location $x_{d}$ versus frequency. - - -, Uncontrolled analytical; - , Controlled analytical; $\Delta$, Controlled experimental. 
in both the uncontrolled and controlled system, also shows the typical jump of $180^{\circ}$ at the poles and zeros. Thus it is clear from Fig. 6 that the controlled system behaves as having new eigenproperties when the error microphone is located such as to render $\alpha_{e}=\beta_{e}=0$. The centered symbols correspond to experimental results for the controlled system and there is very good agreement with the calculated values for excitation frequencies above $200 \mathrm{~Hz}$. The differences found at low frequencies is probably due to the reverberation present in the chamber for excitations below its cutoff frequency of 200 Hz. Nevertheless, the experimental results show the same trend as the analytical curve.

The roots of the polynomial $D(\omega)$ in Eq. (17) were next computed by using the IMSL routine ZPOLR, and they are presented in Table II. As shown in the formulation, the controlled system has one less dynamic degree of freedom than the original beam. Since we have included ten uncontrolled modes in the analysis, there are nine controlled eigenvalues or roots. The last column in Table II lists the natural frequencies of the controlled system $f_{c l}$, and the first five natural frequencies are the resonance condition shown in Fig. 6 by the continuous line. The mode shapes associated to the first five controlled beam natural frequencies were then computed using Eq. (24), and they are shown in Fig. 7(a)-(e) as a continuous line. Note that these mode shapes were not simply obtained by calculating the total system response at the resonance frequencies, but are obtained from individual system characteristic functions. These mode shapes were experimentally determined by driving the controlled system at the frequency of the peaks and measuring the beam response at several axial positions with a movable mini-accelerometer and considering the relative phase with respect to the fixed mini-accelerometer located at the input force location. The measured modes, after being normalized with respect to the uncontrolled beam mass distribution, are plotted in the same Fig. 7(a)-(e) and good agreement with the computed modes is again demonstrated.

By numerical integration, the eigenfunctions of the controlled system can be shown to be nonvolumetric. Since the response is a linear expansion of the modes, this implies that the net volume displaced by the controlled beam is zero at all excitation frequencies. A physical interpretation of this phenomenon is easily described in the wave number domain.

TABLE II. Eigenvalues and natural frequencies of controlled beam.

\begin{tabular}{ccc}
\hline \hline Mode & $\begin{array}{c}\text { Eigenvalue } \\
\omega_{c l}^{2}\left[\mathrm{rad} / \mathrm{s}^{2}\right]\end{array}$ & $\begin{array}{c}\text { Natural frequency } \\
f_{c l}[\mathrm{~Hz}]\end{array}$ \\
\hline 1 & $0.58405 \mathrm{E}+6$ & 121.63 \\
2 & $0.96696 \mathrm{E}+6$ & 156.50 \\
3 & $0.94042 \mathrm{E}+7$ & 488.07 \\
4 & $0.30784 \mathrm{E}+8$ & 883.05 \\
5 & $0.61273 \mathrm{E}+8$ & 1245.82 \\
6 & $0.13691 \mathrm{E}+8$ & 1877.04 \\
7 & $0.25621 \mathrm{E}+9$ & 2547.50 \\
8 & $-0.49259 \mathrm{E}+8$ & $-j 1117.02$ \\
9 & $-0.60269 \mathrm{E}+9$ & $-j 3907.22$ \\
\hline \hline
\end{tabular}
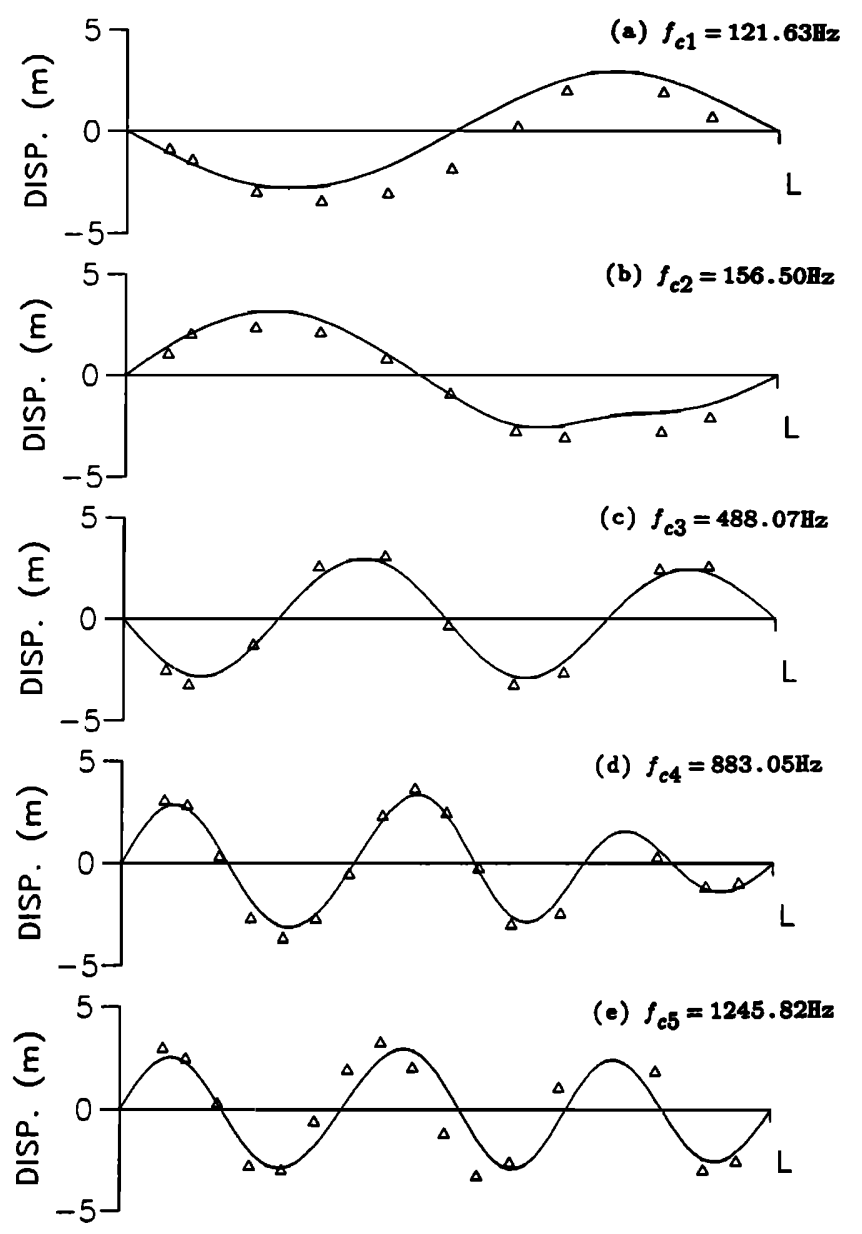

FIG. 7. (a) First, (b) second, (c) third, (d) fourth, and (e) fifth controlled system mode shapes. - , analytical; $\Delta$, experimental.

The sound radiated by a structure is the contribution of all the supersonic structural wave components $k_{s}<k_{0}$ where $k_{s}$ and $k_{0}$ are the structural and acoustic wave numbers, respectively. A structural wave number $k_{s}=0$, that corresponds to the net volume displaced by the structure, will radiate uniformly into the acoustic field at an angle of $\theta=0^{\circ}$. Consequently, this supersonic component is observable by any error microphone located in the acoustic field, and thus it will be canceled by the control input. Then, the wave number spectrum of the controlled system response will be zero at $k_{s}=0$, and thus the net volume of fluid displaced is zero. Since the controlled system response is a linear combination of the modes, each mode shape must be nonvolumetric.

The radiation directivity patterns are given in Figs. 8 and 9 for selected excitation frequencies and normalized for a unit magnitude of the input force $F$. In all figures, the dashed and continuous lines represent the uncontrolled and controlled sound radiated pressure in the $x-z$ plane at a distance of $4.45 L$ computed by Eq. (10), where the modal influence function $\xi_{m}$ used in this analysis is given in Appen$\operatorname{dix} \mathrm{B}$. The negative angle $\theta$ in these plots corresponds to an angle $\psi=180^{\circ}$. The acoustic field was also experimentally measured by the circular array of 13 microphones and these readings are presented as centered circle and triangle sym- 
(a) $f_{1}=279.07 \mathrm{kz}$

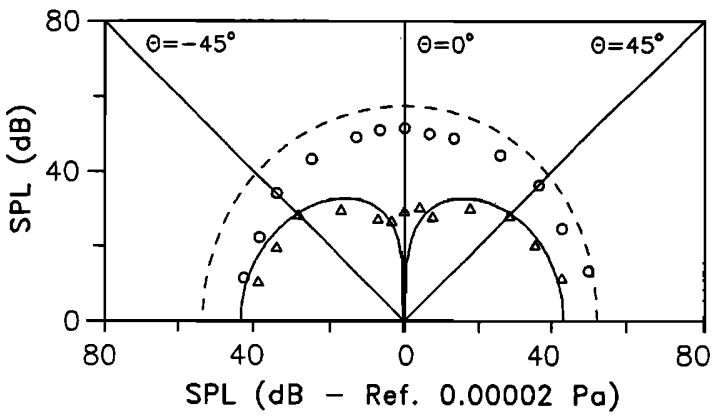

(b) $f_{2}=756.32 \mathrm{~Hz}$

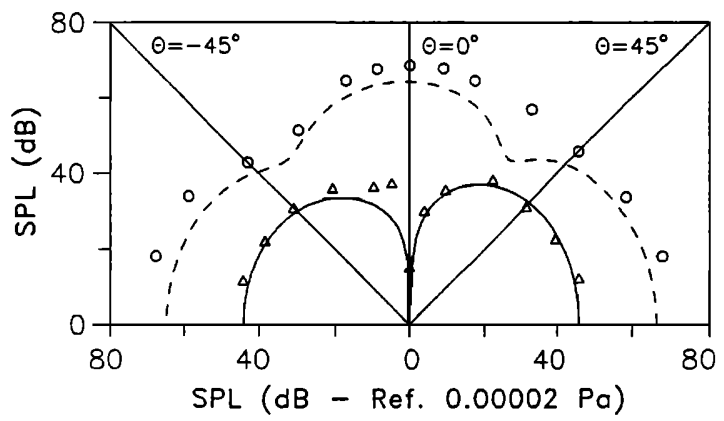

FIG. 8. Radiation directivity pattern for the (a) third and (b) fifth uncontrolled natural frequencies. - - -, analytical uncontrolled; - , analytical controlled; $\bigcirc$, experimental uncontrolled; $\Delta$, experimental controlled.

bols for the controlled and uncontrolled values, respectively. Figure 8 (a) and (b) show the far-field radiation at 279 and $756 \mathrm{~Hz}$, which correspond to the third and fifth resonance frequencies of the uncontrolled beam but off-resonance for the controlled beam. At these frequencies the radiation is not only reduced at the direction of the error microphone $(\theta=0)$ but global attenuation is also achieved. The sound radiation at the first five resonance frequencies of the controlled system in Table II is presented in Fig. 9(a)-(e). The analytical results show the radiated sound pressure in the controlled case (continuous line) to be globally the same or higher than in the uncontrolled case, even though the sound pressure at the error microphone is completely canceled. Good agreement is also observed between the analytical and experimental results except in Fig. 9(a) and (b). These last two plots correspond to the first and second controlled natural frequencies at 121 and $156 \mathrm{~Hz}$, where the test chamber is far from anechoic. However, they are presented for completeness.

\section{CONCLUSIONS}

The dynamic behavior of a SISO feedforward control system in active control of radiation was investigated. When the error microphone is placed along the normal direction with respect to the radiating surface, the controlled system response is then expanded in terms of new mode shapes and natural frequencies. The controlled system eigenvalues are obtained by solving the roots of a polynomial, which is the characteristic function of the controlled system. The associated mode shapes are then computed as a linear combina- (a) $f_{c 1}=121.63 \mathrm{~Hz}$

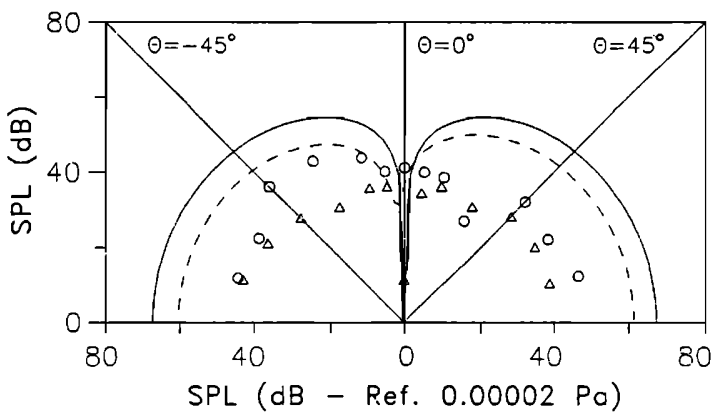

(b) $f_{\mathrm{c} 2}=156.50 \mathrm{~Hz}$

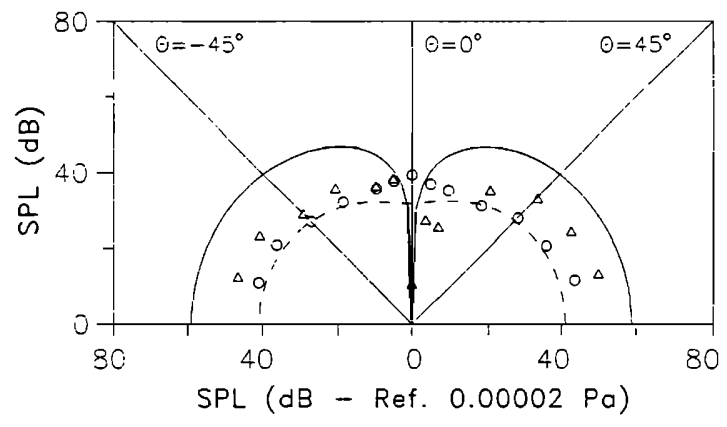

(c) $f_{\mathrm{c} 3}=488.07 \mathrm{~Hz}$

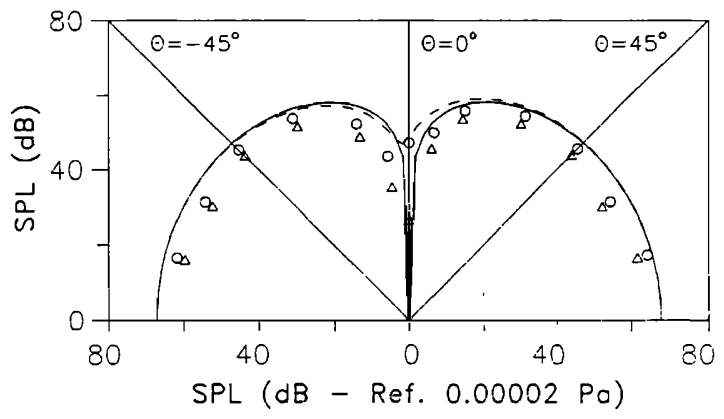

(d) $f_{\mathrm{c} 4}=883.05 \mathrm{~Hz}$

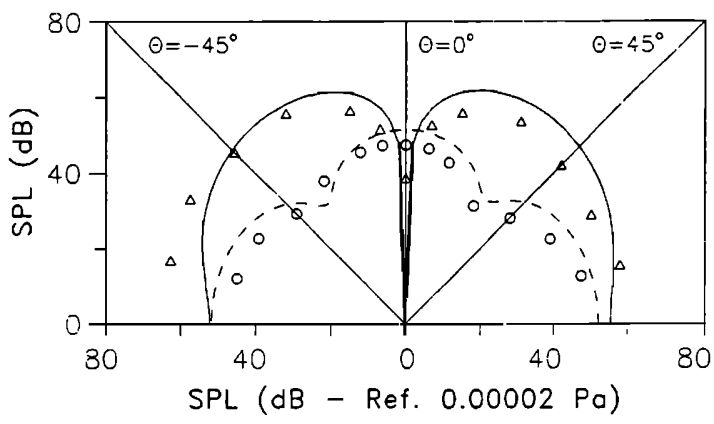

(e) $f_{e 5}=1245.82 \mathrm{Bz}$

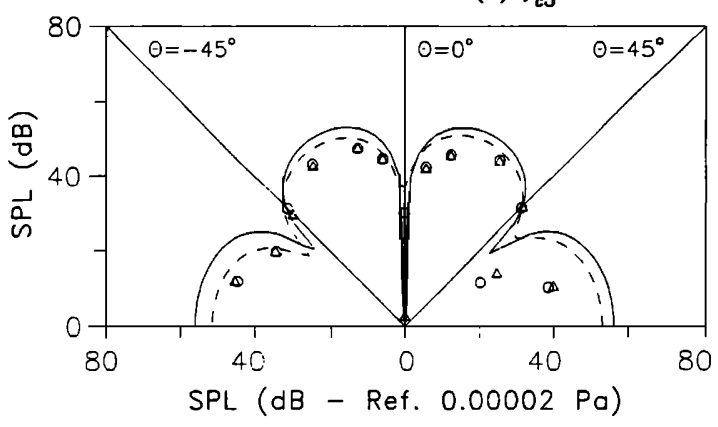

FIG. 9. Radiation directivity pattern for the (a) first, (b) second, (c) third, (d) fourth, and (e) fifth controlled natural frequencies.---, analytical uncontrolled; - , analytical controlled; $O$, experimental uncontrolled; $\Delta$, experimental controlled. 
tion of the uncontrolled modes. Experiments have confirmed the analytical prediction for closed loop modes, their natural frequencies and their associated radiation patterns.

Radiation control from a simply supported beam was analytically and experimentally investigated. The error microphone was placed along a line perpendicular to the beam surface. Global attentuation of the noise field was shown to be only possible at frequencies away from the controlled system natural frequencies. At the natural frequencies of the controlled system, the sound radiation will in fact be increased even though local attenuation at the error microphone is always realizable. This highlights the importance of having a formulation to predict the resonant condition of the controller-beam system. This formulation can also provide the basis for the development of a structural sensor to replace the error microphone.

\section{ACKNOWLEDGMENTS}

The authors gratefully acknowledge the support of this work by DARPA and the Office of Naval Research under Grant No. ONR-00014-88-k-0721.

\section{APPENDIX A}

The derivation of the controlled modal coordinate $q_{n}^{c}(\omega)$ in Eq. (19) is presented in this Appendix.

The controller $G(\omega)$ can be written as

$$
G(\omega)=-\frac{N(\omega)}{D(\omega)}=\frac{\sum_{m=1}^{N} \phi_{m}\left(x_{d}\right) \xi_{m} \Pi_{i, n}^{N}\left(\omega_{s}^{2}-\omega^{2}\right)}{\sum_{m=1}^{N} p_{m} \xi_{m} \Pi_{i \neq n}^{N}\left(\omega_{s}^{2}-\omega^{2}\right)},
$$

where $\xi_{m}$ is a constant quantity. The form that this constant will take depends on the particular problem to be analyzed. Then, the controller becomes the ratio of two polynomials of the same order in the variable $\omega^{2}$. Solving for the $(N-1)$ roots of these polynomials, Eq. (A1) can be written as

$$
G(\omega)=\kappa \frac{\sum_{l=1}^{N-1}\left(\rho_{c l}^{2}-\omega^{2}\right)}{\sum_{l=1}^{N-1}\left(\omega_{c l}^{2}-\omega^{2}\right)},
$$

where

$$
\kappa=\frac{\sum_{m=1}^{N} \phi_{m}\left(x_{d}\right) \xi_{m}}{\sum_{m=1}^{N} p_{m} \xi_{m}} .
$$

The roots of the numerator and denominator in Eq. (A2) are the zeros, $\rho_{c l}$, and poles, $\omega_{c l}$, of the controller. Furthermore, the poles of the controller are also the resonance frequencies of the controlled structure. By using partial fraction expansion, Eq. (A2) can be put in the form

$$
\begin{aligned}
G(\omega) & =\frac{\kappa}{N-1} \sum_{l=1}^{N-1} \frac{\left(A_{l}-\omega^{2}\right)}{\left(\omega_{c l}^{2}-\omega^{2}\right)} \\
& =\frac{\kappa}{N-1} \sum_{l=1}^{N-1}\left(A_{l}-\omega^{2}\right) H_{c l}(\omega) .
\end{aligned}
$$

The partial fraction coefficients $A_{l}$ can be easily computed. However, it will be shown later that their computation is not required. The controller in Eq. (A4) can now be replaced into Eq. (13) and this in turn into Eq. (5) to give

$$
\begin{aligned}
q_{n}^{c}(\omega)= & \left(\phi_{n}\left(x_{d}\right) H_{n}(\omega)\right. \\
& \left.-\frac{p_{n} \kappa}{N-1} \sum_{l=1}^{N-1}\left(A_{l}-\omega^{2}\right) H_{c l}(\omega) H_{n}(\omega)\right) F,
\end{aligned}
$$

where the product of the FRFs $H_{n}(\omega) H_{c l}(\omega)$ can be further solved in partial fractions as follows

$$
\left(A_{l}-\omega^{2}\right) H_{n}(\omega) H_{c l}(\omega)=C_{l n} H_{c l}(\omega)+D_{l n} H_{c l}(\omega),
$$

where

$$
\begin{aligned}
& C_{l n}=\left(\omega_{c l}^{2}-A_{l}\right) /\left(\omega_{c l}^{2}-\omega_{n}^{2}\right), \\
& D_{l n}=\left(A_{l}-\omega_{n}^{2}\right) /\left(\omega_{c l}^{2}-\omega_{n}^{2}\right) .
\end{aligned}
$$

Replacing Eq. (A6) into (A5) and reordering terms gives

$$
\begin{aligned}
q_{n}^{c}(\omega)= & {\left[\left(\phi_{n}\left(x_{d}\right)-\frac{p_{n} \kappa}{N-1} \sum_{l=1}^{N-1} D_{l n}\right) H_{n}(\omega)\right.} \\
& \left.-\frac{p_{n} \kappa}{N-1} \sum_{l=1}^{N-1} C_{l n} H_{c l}(\omega)\right] F .
\end{aligned}
$$

Evaluating Eq. (13) at $\omega=\omega_{n}$, it is easy to show that

$$
G\left(\omega_{n}\right)=\frac{\phi_{n}\left(x_{d}\right)}{p_{n}}=\frac{\kappa}{N-1} \sum_{l=1}^{N-1} D_{l n} .
$$

Substituting Eq. (A9) into (A8) gives the controlled modal coordinate as

$$
\begin{aligned}
q_{n}^{c}(\omega) & =\sum_{l=1}^{N-1}\left(-\frac{p_{n} \kappa}{N-1} C_{l n}\right) H_{c l}(\omega) F \\
& =\sum_{l=1}^{N-1} \gamma_{l n} H_{c l}(\omega) F
\end{aligned}
$$

that is also Eq. (19).

\section{APPENDIX B}

The computation of the eigenvalues $\omega_{n}^{2}$ and eigenfunctions $\phi_{n}(x)$ of the beam including the concentrated mass and end torsional springs is presented in this Appendix. The assumed-mode method ${ }^{15}$ is used to solve the eigenvalue problem. The mode shapes of the uniform simply supported beam

$$
\psi_{i}(x)=\left(\frac{2}{m L}\right)^{1 / 2} \sin \left(\frac{i \pi x}{L}\right)
$$

are selected as admissible functions. Then, the response of the beam is expanded as

$$
w(x, t)=w(x) e^{j \omega t}=\left(\sum_{i=1}^{N} \psi_{i}(x) a_{i}\right) e^{j \omega t},
$$

where $a_{i}$ are expansion coefficients. Then, Eq. (B2) is used to compute the strain and kinetic energy of the system as follows:

$$
\begin{aligned}
V= & \frac{1}{2} \int_{0}^{L} E I\left(w_{, x x}\right)^{2} d x \\
& +\frac{1}{2} K\left(w_{, x}\right)_{x=0}^{2}+\frac{1}{2} K\left(w_{, x}\right)_{x=L}^{2}
\end{aligned}
$$

and 


$$
T=\frac{1}{2} \int_{0}^{L} m\left(w_{, t}\right)^{2} d x+\frac{1}{2} M\left(w_{, t}\right)_{x=x_{d}}^{2}
$$

where $V$ is the strain energy, $T$ is the kinetic energy, $K$ is the spring constant, and $M$ is the concentrated mass. Replacing Eq. (B2) into Eqs. (B3) and (B4) and applying Lagrange's equations, ${ }^{15}$ the eigenvalue problem can be written as follows:

$$
\mathbf{K} \underline{a}=\omega^{2} \mathbf{M} a,
$$

where $\mathbf{K}$ and $\mathbf{M}$ are the stiffness and mass matrices of the system, and $a=\left\{a_{1}, \ldots, a_{N}\right\}^{T}$. The dimension of these matrices is $N \times N$ and their elements are given by

$$
\begin{aligned}
& k_{r s}=\delta_{r s} \omega_{r}^{2}+K\left[\psi_{r, x}(0) \psi_{s, x}(0)+\psi_{r, x}(L) \psi_{s, x}(L)\right], \\
& m_{r s}=\delta_{r s}+M \psi_{r}\left(x_{d}\right) \psi_{s}\left(x_{d}\right),
\end{aligned}
$$

and

$$
\omega_{r}^{2}=\left(\frac{r \pi}{L}\right)^{4} \frac{E I}{m}
$$

is the eigenvalue of the uniform simply supported beam.

Solving the eigenproblem in Eq. (B4), there will be $N$ eigenvalues $\omega_{n}^{2}$ and associated eigenvectors $\underline{a}_{n}=\left\{a_{1 n}, \ldots\right.$, $\left.a_{N n}\right\}^{T}$. Then, the eigenfunctions of the continuous beam are finally obtained as

$$
\phi_{n}(x)=\sum_{i=1}^{N} \psi_{i}(x) a_{i n}
$$

The modal acoustic influence function $\xi_{m}$ used in the numerical analysis can now be derived. The modal acoustic influence function is obtained from Eq. (8) by setting $\alpha=\beta=0$. Then,

$$
\xi_{n}=\int_{0}^{b} \int_{0}^{L} \phi_{n}(x) d x d y
$$

Replacing Eqs. (B1) and (B9) into (B10) gives

$$
\xi_{n}=\int_{0}^{b} \int_{0}^{L} \sum_{i=1}^{N}\left(\frac{2}{m L}\right)^{1 / 2} \sin \left(\frac{i \pi x}{L}\right) a_{i n} d x d y
$$

or

$$
\xi_{n}=b L\left(\frac{1}{m L}\right)^{1 / 2} \sum_{i=1}^{N} a_{i n} \frac{1-(-1)^{i}}{i \pi}
$$

${ }^{1}$ G. E. Warnaka, "Active Attenuation of Noise-The State of the Art," Noise Control Eng. 18(3), 100-110 (1982).

2J. E. Ffowcs-Williams, “Anti-sound,” Proc. R. Soc. London, Ser. A 395, 63-88 (1984).

${ }^{3}$ C. Deffayet and P. A. Nelson, "Active Control of Low Frequency Harmonic Sound Radiated by a Finite Panel," J. Acoust. Soc. Am. 84(6), 2192-2199 (1988).

${ }^{4}$ C. R. Fuller, “Apparatus and methods for global noise reduction," U.S. Patent No. 4,715,599 (1987)

${ }^{5}$ C. R. Fuller, "Active Control of Sound Transmission/Radiation from Elastic Plates by Vibration Inputs--I. Analysis," J. Sound Vib. 136(1), 115 (1990).

${ }^{6}$ V. L. Metcalf, C. R. Fuller, R. J. Silcox, and D. E. Brown, “Active Control of Sound Transmission/Radiation from Elastic Plates by Vibration Inputs-II Experiments," J. Sound Vib. 153(3), 387-402 (1992).

${ }^{7}$ C. R. Fuller, C. H. Hansen, and S. D. Snyder, "Experiments on Active Control of Sound Radiation Using a Piezoceramic Actuator," J. Sound Vib. 150(2), 179-190 (1991).

${ }^{8}$ R. L. Clark and C. R. Fuller, "Experiments on Active Control of Structurally Radiated Sound Using Multiple Piezoceramic Actuators," J. Acoust. Soc. Am. (accepted for publication) (1990).

${ }^{9}$ R. A. Burdisso and C. R. Fuller, "Theory of Feedforward Controlled System Eigenproperties," J. Sound Vib. 153(3), 437-452 (1992).

${ }^{10}$ R. A. Burdisso and C. R. Fuller, "Eigenproperties of Feedforward Controlled Flexible Structures," in Recent Advances in Active Control of Sound and Vibration (Technomic, Lancaster, 1991), pp. 851-863.

"S. Burke and J. Hubbard, "Distributed Parameter Control Design for Vibrating Beams Using Generalized Functions," in Proceedings of the 4th IFAC Symposium on the Control of Distributed Parameter Systems, Los Angeles, CA (Pergamon, New York, 1987), pp. 161-166.

${ }^{12} \mathrm{M}$. Obal, "Vibration Control of Flexible Structures Using Piezoelectric Devices as Sensors and Actuators," Ph.D. Thesis, School of Aerospace Engineering, Georgia Institute of Technology (1986).

${ }^{13}$ E. K. Dimitriadis, C. R. Fuller, and C. R. Rogers, "Piezoelectric Actuators for Distributed Vibration Excitation of Thin Plates," J. Vib. Acoust. 113, 100-107 (1989).

${ }^{14}$ F. Fahy, Sound and Structural Vibration (Academic, Orlando, FL, 1985).

${ }^{15}$ C. E. Wallace, "Radiation Resistance of a Baffled Beam," J. Acoust. Soc. Am. 51, 936-945 (1972)

${ }^{16}$ C. E. Wallace, "Radiation Resistance of a Rectangular Panel," J. Acoust. Soc. Am. 51, 946-952 (1972).

${ }^{17}$ R. R. Craig Jr., Structural Dynamics (Wiley, New York, 1981).

${ }^{18}$ B. Widrow, and S. D. Stearns, Adaptive Signal Processing (Prentice-Hall, New York, 1985).

${ }^{19}$ S. J. Elliot, I. M. Stothers, and P. A. Nelson, "A Multiple Error LMS Algorithm and Its Application to the Active Control of Sound and Vibration," IEEE Trans. Acoust. Speech Signal Process. ASSP-35(10), 14231434 (1987). 